\title{
Corrigendum
}

\section{Blocking p55PIK signaling inhibits proliferation and induces differentiation of leukemia cells}

\author{
G Wang, Y Deng, X Cao, S Lai, Y Tong, X Luo, Y Feng, X Xia, J Gong and J Hu \\ Cell Death and Differentiation (2014) 21, 1664; doi:10.1038/cdd.2014.107
}

Correction to: Cell Death and Differentiation (2012) 19, 1870-1879; doi:10.1038/cdd.2012.70; published online 22 June 2012

Since the publication of this paper the authors have noticed there were errors in two series of letters in the Materials and Methods section. The correct paragraph is shown below. The authors would like to apologize for any inconvenience caused.

Expression and purification of TAT-N24 and control TAT-N24M fusion proteins. CDNA fragments encoding
6xHis tag, PDT in Tat protein (YGRKKRRQRRR), HA tag (YPYDVPDYA) and N24 (MDRDDADWREVMMPYSTELIFYIE) were introduced into pMD18-T Easy vector (Invitrogen, Grand Island, NY, USA). His-TAT-HA-N24 cDNA fragment then was subcloned into the vector pET32a (Invitrogen). To generate the control fusion protein (TAT-N24M), the N24 cDNA sequence was replaced by cDNA encoding the shuffled N24 (MDRDDWDAREVIMMPYSELYTIFE). The TAT-N24 and TAT-N24M fusion proteins have 96 amino-acid residues. The obtained recombinant vectors (designated pHisTAT-N24 and pHisTAT-N24M) were transformed into $E$. coli BL21 (DE3) for fusion protein expression. 